\title{
Breast Cancer Survival at a Leading Cancer Centre in Malaysia
}

\author{
Matin Mellor Abdullah ${ }^{1 *}$, Ahmad Kamal Mohamed ${ }^{1}$, Yoke Ching Foo ${ }^{1}$, Catherine \\ May Ling Lee ${ }^{1}$, Chin Teong Chua², Chin Huei $\mathrm{Wu}^{3}$, LP Hoo ${ }^{4}$, Teck Onn $\mathrm{Lim}^{4}$, \\ Sze Whey Yen ${ }^{5}$
}

\begin{abstract}
Background: GLOBOCAN12 recently reported high cancer mortality in Malaysia suggesting its cancer health services are under-performing. Cancer survival is a key index of the overall effectiveness of health services in the management of patients. This report focuses on Subang Jaya Medical Centre (SJMC) care performance as measured by patient survival outcome for up to 5 years. Materials and Methods: All women with breast cancer treated at SJMC between 2008 and 2012 were enrolled for this observational cohort study. Mortality outcome was ascertained through record linkage with national death register, linkage with hospital registration system and finally through direct contact by phone or home visits. Results: A total of 675 patients treated between 2008 and 2012 were included in the present survival analysis, $65 \%$ with early breast cancer, $20 \%$ with locally advanced breast cancer (LABC) and $4 \%$ with metastatic breast cancer (MBC). The overall relative survival (RS) at 5 years was $88 \%$. RS for stage I was $100 \%$ and for stage II, III and IV disease was $95 \%, 69 \%$ and $36 \%$ respectively. Conclusions: SJMC is among the first hospitals in Malaysia to embark on routine measurement of the performance of its cancer care services and its results are comparable to any leading centers in developed countries.
\end{abstract}

Keywords: Breast cancer - cancer survival - cancer performance measurement - cohort study

Asian Pac J Cancer Prev, 16 (18), 8513-8517

\section{Introduction}

Cancer is a leading cause of death in the world, and a widening disparity has emerged between developed and developing countries. GLOBOCAN12 recently reported high cancer mortality in Malaysia, a developing country, suggesting its cancer health services is under-performing though there are likely individual centres of excellence such as the Subang Jaya Medical Centre, Malaysia (SJMC).

The aims of healthcare are to provide services that are safe, effective, patient centred and of value. Healthcare performance measurement then evaluates the extent to which the health services rendered to patients meet these aims. These measurements are intended to serve accountability purposes and to promote improvements in the delivery of care. Healthcare Performance Measurement and Reporting system [(HPMRS) more details at www. hpmrs.com.my] is the local statistical system developed to meet the increasing demands for healthcare performance measurement services.

SJMC's cancer care services, and specifically breast cancer care, is the focus of this report. We have previously reported on SJMC's breast cancer care performance for process measures (Abdullah et al, 2014). The present report focuses on SJMC's care performance as measured by patient survival outcome for up to 5 years. Cancer survival is a key index of the overall effectiveness of health services in the management of patients with cancer. Persistent difference in survival between a centre's performance and a reference population's or benchmark results represents many avoidable deaths.

\section{Materials and Methods}

We conducted a single-centre, observational cohort study to estimate the survival outcome of patients diagnosed in SJMC between 2008 and 2012. The Ministry of Health's Medical and Research Ethics Committee approved the study protocol.

\section{Study population}

The study population consisted of Malaysian women with pathologically confirmed primary breast cancer diagnosed between 2008 and 2012, and treated with at least one treatment modality at SJMC. Cases were identified through the hospital register as well as operative surgery, chemotherapy and radiotherapy records. Case 
ascertainment was independently verified to be complete $(100 \%)$. Foreign patients, patients with non-epithelial malignancy or recurrent tumour were excluded from analysis.

\section{Data collection and definitions}

At enrolment, data were abstracted from patients' medical and histopathology (HPE) reports by trained data collectors. Demographic data abstracted included age, sex, race and nationality; tumour characteristics included histologic type, grade, location, extent, and size; lymph node and distant organ metastases. Staging of disease was based on the American Joint Committee on Cancer (AJCC) criteria. AJCC stage I or II disease were considered early breast cancer (EBC), stage III locally advanced breast cancer (LABC) and stage IV metastatic breast cancer (MBC).

After enrolment, all patients were followed up for 12 months to collect data on their subsequent exposure to cancer-directed therapies, which were abstracted from medical, operative surgery, chemotherapy and radiotherapy records.

For the purpose of measuring breast cancer care performance, we mostly adopted the performance measures developed and used by Quality Oncology Practice Initiative (QOPI) (Campina et al, 2011; American Society of Clinical Oncology, 2012), American Society of Clinical Oncology/National Comprehensive Cancer Network (ASCO- NCCN) (American Society of Clinical Oncology, 2007; Desch et al, 2008) and, National Accreditation Program for Breast Centres (NAPBC) (National Accreditation Program for Breast Centres, 2012), while taking into account local clinical practice guideline (Ministry of Health Malaysia, 2010).

\section{Mortality ascertainment and imputation}

Complete and accurate ascertainment of mortality outcome among study patients is necessary to minimize bias in estimating cancer survival outcome. We followed a rigorous procedure described below to ensure this. $i$ ). Case ascertainment was initially independently verified to be complete (100\%). This was to avoid exclusion of deceased patients especially those who die soon after diagnosis. ii). Mortality outcomes were noted during data abstraction for the study (six deaths identified). iii). All cases enrolled were matched twice in 2013 and 2014, based on their names and national identity card number against the mortality database provided by the National Registration Department to ascertain their mortality outcome (total 41 deaths identified). $i v$ ). Remaining cases were matched based on their names and hospital number against the hospital register (which records all visits to the hospital). Patients who had a visit after the end of the study period (31 Dec 2013) were considered alive (403 ascertained alive). $v$ ). A sample of the remaining cases with Stage I or II or no staging information and 100\% of cases with Stage III or IV were contacted by phone or home visit to ascertain mortality outcomes. All patients with Stage I and no staging information were alive. One (3\%) patient out of 32 with Stage II had died, likewise for five (12\%) out of 42 Stage III and two (40\%) out of six
Stage IV patients. vi). For the purpose of survival analysis, we therefore assumed all cases with Stage I or no staging information that were not contacted (60 cases), to be alive. For the 43 cases with Stage II, we randomly selected one case and imputed her outcome as death. We assumed all remaining uncontacted patients with Stage III (six cases) and IV (one case) to be dead. Thus, any bias in the survival estimates arising from missing information on mortality outcome is conservative (that is, the survival estimates can only be worse than they actually are).

\section{Independent data audit}

HPE reports were retrieved for all patients enrolled to verify tumor diagnosis and characteristics. In addition, patients' demographic and treatment data were also subjected to independent data verification against source documents on site. The accuracy of the collected data with respect to demographics, surgery, radiotherapy, chemotherapy, hormonal therapy and trastuzumab treatment were all $>95 \%$.

\section{Statistical methods}

Continuous variables are described by summary statistics such as mean, median, and standard deviation and categorical (nominal/ordinal) variables, by the frequencies of each category.

For cancer survival outcome performance, results are expressed as overall survival and relative survival. Relative survival is the ratio of the survival observed in the study patients and the survival that would be expected if they had experienced only the background mortality (all-cause death rates) of the general population of the same age, sex and ethnicity. It shows the extent to which cancer shortens life compares to the general population.

Age standardised five-year relative survival is used for comparison of survival outcome between this study population and other centres' or registry populations. Age standardised rate refers to the rate that would be observed if the patient populations compared had the same age structure as an external standard population, in this case, the International Cancer Survival Standard (Corazziari et al, 2004). Age standardisation allows comparison of results between jurisdictions or countries. Multivariable Cox regression is used to estimate the effects of covariates on survival outcome. The level of significance was set at 0.05 .

\section{Results}

A total of 836 patients who were potentially eligible for inclusion in this study were identified to have breast cancer through the hospital register as well as operative surgery, chemotherapy and radiotherapy records. Ninety patients were excluded because of incomplete data (34 uncertain date of diagnosis, 15 no pathology reports confirming cancer diagnosis, 41 no treatment details). A further 71 patients were excluded because of non-eligibility (nonprimary tumour 50 , non- epithelial tumour 10 , foreign patients 14). Thus the final sample size was 675 subjects.

Baseline characteristics of breast cancer patients, SJMC 
Table 1. Patient and Tumour Characteristics at Diagnosis

\begin{tabular}{|c|c|c|}
\hline Patient characteristics & Statistics & Results \\
\hline Number of patients & Numbers & $675(100 \%)$ \\
\hline \multirow[t]{3}{*}{ Age, years } & Mean (SD) & $53(11)$ \\
\hline & Median (IQR) & $53(46,59)$ \\
\hline & $(\operatorname{Min}, \operatorname{Max})$ & $(24,88)$ \\
\hline \multirow[t]{4}{*}{ Age distribution } & No. $(\%)$ Age $<40$ & $57(8)$ \\
\hline & No. (\%) Age 40 to 49 & $190(28)$ \\
\hline & No. (\%) Age 50 to 59 & $260(39)$ \\
\hline & No. $(\%)$ Age $>=60$ & $168(25)$ \\
\hline \multirow[t]{2}{*}{ Sex } & No. (\%) Male & $6(1)$ \\
\hline & No $(\%)$ Female & $669(99)$ \\
\hline \multirow[t]{5}{*}{ Race } & No. (\%) Malay & $92(14)$ \\
\hline & No. (\%) Chinese & $523(77)$ \\
\hline & No. $(\%)$ Indian & $54(8)$ \\
\hline & No. (\%) Bumiputera Sabah & $1(0)$ \\
\hline & No. $(\%)$ Others & $5(1)$ \\
\hline \multirow[t]{4}{*}{ Healthcare funding } & No. (\%) Private out-of-pocket & $577(85)$ \\
\hline & No. (\%) Private insurance & $59(9)$ \\
\hline & No. (\%) Employers & $38(6)$ \\
\hline & No. (\%) Missing & $1(0)$ \\
\hline \multirow[t]{4}{*}{ Residence } & No. (\%) Klang Valley & $494(73)$ \\
\hline & No. (\%) Selangor & $33(5)$ \\
\hline & No. (\%) Outside Selangor & $63(9)$ \\
\hline & No. (\%) Missing & $85(13)$ \\
\hline \multirow[t]{2}{*}{ Origin } & No. (\%) Local & $304(45)$ \\
\hline & No. $(\%)$ Referral & $371(55)$ \\
\hline \multirow[t]{4}{*}{ Duration from first presentation to diagnosis, in days* } & Number of patients first presenting at SJMC & 304 \\
\hline & Mean (SD) & $7(7)$ \\
\hline & Median (IQR) & $6(6,6)$ \\
\hline & $(\operatorname{Min}, \operatorname{Max})$ & $(0,35)$ \\
\hline \multirow[t]{4}{*}{ Stage at diagnosis } & No. (\%) Early Breast Cancer (EBC) & $440(65)$ \\
\hline & No. (\%) Locally Advanced Breast Cancer (LABC) & $134(20)$ \\
\hline & No. (\%) Metastatic Breast Cancer (MBC) & $28(4)$ \\
\hline & No. $(\%)$ without staging information & $73(11)$ \\
\hline \multirow[t]{4}{*}{ Tumour size } & No. $(\%) \mathrm{T} 1$ (1 to $20 \mathrm{~mm})$ & $167(25)$ \\
\hline & No. $(\%) \mathrm{T} 2$ (21 to $50 \mathrm{~mm})$ & $179(27)$ \\
\hline & No. $(\%) \mathrm{T} 3(>50 \mathrm{~mm})$ & $15(2)$ \\
\hline & No. $(\%)$ Unknown & $314(47)$ \\
\hline \multirow[t]{5}{*}{ Regional node } & No. (\%) Negative node & $160(24)$ \\
\hline & No. (\%) 1-3 Positive node & $48(7)$ \\
\hline & No. (\%) 4-10 Positive node & $56(8)$ \\
\hline & No. $(\%)>10$ Positive node & $32(5)$ \\
\hline & No. (\%) Unknown & $379(56)$ \\
\hline \multirow[t]{3}{*}{ Tumour histology } & No. (\%) Invasive breast carcinoma & $518(77)$ \\
\hline & No. (\%) Ductal carcinoma in situ & $29(4)$ \\
\hline & No. $(\%)$ Other carcinomas & $128(19)$ \\
\hline \multirow[t]{4}{*}{ Grading } & No. (\%) Grade 1 & $33(5)$ \\
\hline & No. $(\%)$ Grade 2 & $162(24)$ \\
\hline & No. (\%) Grade 3 & $141(21)$ \\
\hline & No. (\%) No information & $339(50)$ \\
\hline \multirow[t]{11}{*}{ Biomarkers } & No. $(\%)$ ER+ & $461(72)$ \\
\hline & No. (\%) No information on ER & 31 \\
\hline & No. $(\%) \mathrm{PR}+$ & $399(63)$ \\
\hline & No. (\%) No information on PR & 40 \\
\hline & No. $(\%) \mathrm{ER}+$ or PR+ & $471(73)$ \\
\hline & No. (\%) No information on ER and PR & 30 \\
\hline & No. (\%) HER2 ISH+ or IHC+ if ISH missing or unknown & $189(34)$ \\
\hline & No. (\%) No information on HER2 & 115 \\
\hline & No. (\%) Triple positive (ER+, PR+, HER+) & $87(16)$ \\
\hline & No. (\%) Triple negative (ER-, PR-, HER-) & $74(13)$ \\
\hline & No. (\%) No information on ER, PR, and/or HER & 123 \\
\hline
\end{tabular}

2008-2012

Table 1 shows the patients' demographic and tumour characteristics. The mean age was only 53 years; $36 \%$ was aged $<50$ years, $77 \%$ was Chinese and $78 \%$ resided in Klang Valley or Selangor. 85\% paid for their care outof-pocket (OOP) and only 14\% had their care financed 
Matin Mellor Abdullah et al

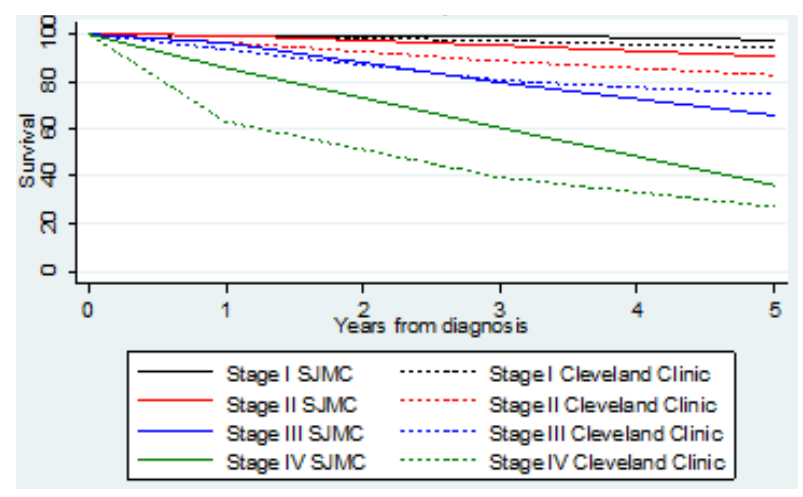

Figure 1. Comparrative Performance between SJMC and Cleveland Clinic in terms of Overall Survival Outcome of Patients with Breast Cancer

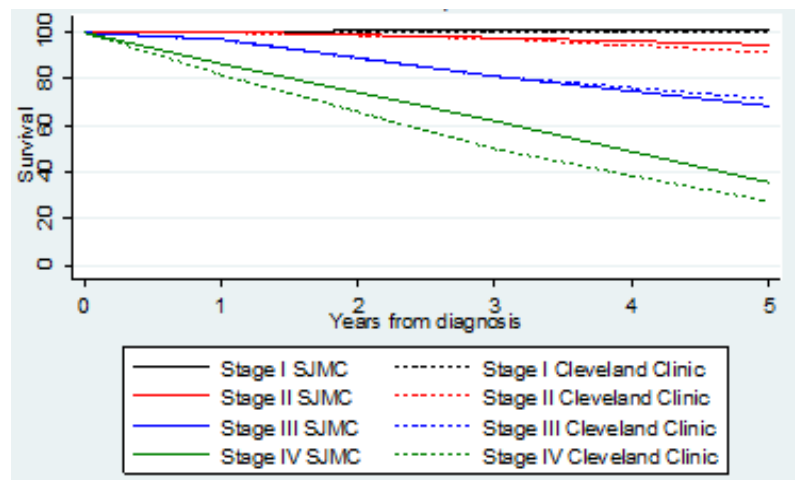

Figure 2. Comparrative Performance between SJMC and Cleveland Clinic in terms of Relative Survival Outcome of Patients with Breast Cancer

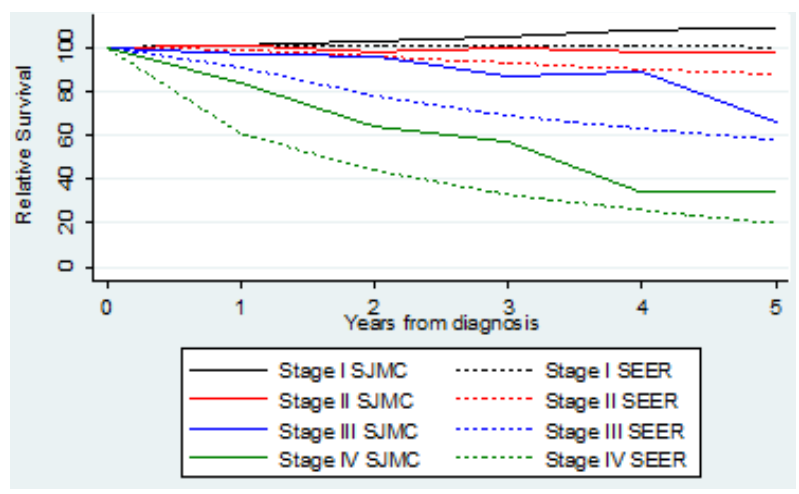

Figure 3. Comparrative of Age Stansardized Five-Year Relative Survival between Patients Treates at SJMC and SEERS

by insurance or their employer.

For patients first presenting to SJMC only, it took a median of 6 days to arrive at a diagnosis of breast cancer. $65 \%$ of patients were diagnosed with EBC (Stage 1 or 2), another $20 \%$ with $\mathrm{LABC}$ and $4 \%$ with late stage metastatic cancer. $25 \%$ had T1 tumour and $24 \%$ were node negative. $72 \%$ were $\mathrm{ER}+, 63 \% \mathrm{PR}+, 34 \% \mathrm{HER} 2+$ and $13 \%$ triple negative.

Treatment for breast cancer, SJMC 2008-2012

Of the 675 patients treated at SJMC between 2008

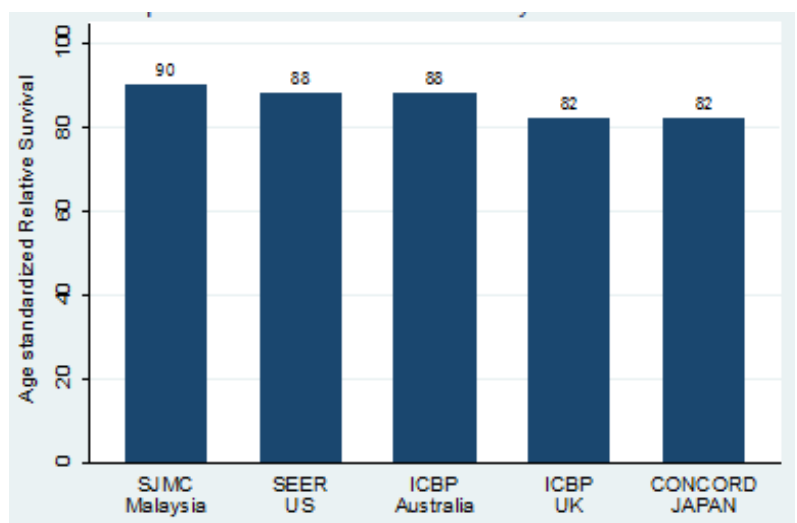

Figure 4. Intemational Comparisons of Breast Cancer Care Performance in Terms of Five Year RelaTive Survival

and 2012, $553(82 \%)$ patients had surgery there but only $30 \%$ of these were breast conserving surgery. 346 (51\%) patients had radiotherapy and 307 (66\%) had chemotherapy. $346(73 \%)$ of $471 \mathrm{ER}+$ or PR+ patients had hormonal therapy and $42(22 \%)$ of 189 HER2+ patients received trastuzumab treatment.

\section{Breast cancer survival outcome performance}

Overall survival at 5 years was $98 \%$ for patients with Stage I disease, decreasing to $36 \%$ for Stage IV disease. More impressively, the relative survival at 5 years was $101 \%$ indicating these patients were practically cured of their cancers. For Stage II disease, the result was 95\%, which is no less remarkable. These results show that SJMC has accomplished similar if not better results than established centres of excellence such as the Cleveland Clinic.

Results on age standardised relative survival at 5 years are available from Surveillance, Epidemiology and End Results (SEER) database, an often used reference population in cancer epidemiologic research. For all cancer stages, SJMC results are clearly superior to the average results accomplished by all cancer centres in the United States, from which SEERS registry population is drawn.

Comparing with the results reported by other countries' registries, SJMC's relative survival results are among the highest. Of course it is not meaningful to compare the result of a single institution (SJMC) directly with the average result reported by a cancer registry for a population. However, in so far that SJMC's results are above the average reported by these registries, it is reasonable to infer that its results match those of the better performing institutions reporting data to those registries.

\section{Discussion}

Malaysia cancer care performance has been previously reported by Lim (2014). This study noted that performance results are probably acceptable for a middle income country though far below the $95 \%$ or higher adherence rates routinely reported by centres in developed countries. A population based retrospective cohort study reported the overall 5-year survival rate was $49 \%$ with median 
survival time of 68.1 months (Abdullah et al, 2013), whilst Ibrahim (2012) also reported that the overall survival rate of Malaysian women with breast cancer was lower than the western figures. Meanwhile, Taib (2011) reported 5-year observed survival improvements from $58.4 \%$ to $75.7 \%$.

CONCORD publication reported age-standardised 5 -year net survival from breast cancer was rates as $68 \%$ for Malaysia based on Penang Cancer Registry data (a regional registry in Malaysia). In the same report, for women diagnosed during 2005-09, age-standardised 5 -year net survival from breast cancer was $80 \%$ or higher in 34 countries around the world (Allemani et al, 2015).

SJMC is among the first hospitals in Malaysia to embark on routine measurement of the performance of its cancer care services. SJMC's cancer care process performance results have been consistently about $90 \%$, this is close to the benchmark of $95 \%$. Not surprisingly the consistently high performing cancer care system in SJMC has translated into excellent patient survival outcomes, the key index of the effectiveness of cancer care services in the management of patients with cancer.

Patients treated at SJMC between 2008 and 2012 had a relative survival at 5-year of $101 \%$ for Stage I disease. This means all such patients were cured by the treatment they had received at SJMC. Even for Stage IV disease, SJMC's result was a respectable $36 \%$.

\section{References}

Abdullah MM, Mohamed AK, Foo YC, et al (2014). P0121 Cancer care performance and survival outcomes at a private cancer centre in Malaysia. Eur J Cancer, 50, 43.

Abdullah NA, Mahiyuddin WRW, Muhammad NA, et al (2013). Survival rate of breast cancer patients in Malaysia: a population-based study. Asian Pac J Cancer Prev, 14, 4591-4.

Allemani C, Weir HK, Carreira H, et al (2015). Global surveillance of cancer survival 1995-2009: analysis of individual data for 25676887 patients from 279 populationbased registries in 67 countries (CONCORD-2). Lancet, 385, 977-1010.

ASCO-NCCN Quality Measures for Breast and Colorectal Cancer Care.

Campion FX, Larson LR, Kadlubek PJ et al (2011). Advancing performance measurement in oncology: quality oncology practice initiative (QOPI) participation and quality outcomes. J Oncol Pract, 7, 31-5.

Corazziari I, Quinn MJ, Capocaccia R (2004). Standard cancer patient population for age standardizing survival ratios. Eur J Cancer, 40, 2307-16.

Desch CE, McNiff KK, Schneider EC, et al (2008). American society of clinical oncology/national comprehensive cancer network quality measures. J Clin Oncol, 26, 3631-7.

GCC Lim, EN Aina, GF Ho, et al (2014). Closing the cancer divide: performance of breast cancer care services in a middle income developing country malaysia.BMC Cancer, 14, 212.

Ibrahim NI, Dahlui M, Aina EN,Al-Sadat N (2012). Who are the Breast Cancer Survivors in Malaysia? Asian Pac J Cancer Prev, 13, 2213-8.

Ministry of Health and Academy of Medicine Malaysia. Clinical Practice Guidelines: Management of Breast Cancer $2^{\text {nd }}$ edition. 2010.

National Accreditation Program for Breast Centers (NAPBC).
Breast Center Standards Manual 2012.

QOPI Quality Measures. Available at: http://qopi.asco.org/ Documents/QOPISpring2012MeasuresSummary_000.pdf

Taib NA, Akmal MN, Mohamed I, Yip CH (2011). Improvement in survival of breast cancer patients - trends in survival over two time periods in a single institution in an Asia Pacific Country, Malaysia. Asian Pac J Cancer Prev, 12, 345-9. 